\title{
PRAGMÁTICA Y ARQUITECTURA MENTAL: UNA PROPUESTA DESDE LA MODULARIDAD MASIVA DE LA MENTE
}

\author{
Nicolás Acuña Luongo \\ Centro Interdisciplinario de Innovación Educativa, Facultad de Educación, \\ Universidad Santo Tomás, Chile. \\ Dirección: Pasaje Los Peumos 1860. Talca \\ Este trabajo fue apoyado por la Agencia Nacional de Investigación y Desarrollo (ANID). \\ Proyecto FONDECYT No 3210639 \\ acuna.nicolas17agmail.com
}

\begin{abstract}
Resumen
Uno de los problemas fundamentales respecto de la naturaleza cognitiva de la pragmática se refiere al lugar que ocupa ella en la arquitectura de la mente. Al respecto existen varias propuestas. Una de ellas sostiene que forma parte de los sistemas centrales en el marco de la teoría de modular de Jerry Fodor. Sin embargo, Asa Kasher plantea una hipótesis basada en la arquitectura modula de Fodor, pero sí otorga un carácter modular al menos a algunos aspectos de la interpretación pragmática. En este trabajo sostenemos que ambas propuestas son incorrectas debido a inconsistencias con los hallazgos empíricos sobre procesamiento pragmático. Por el contrario, se aboga por una hipótesis basada en la modularidad masiva de la mente, en donde consideraciones evolutivas llevaron a que emerja un submódulo pragmático que es parte de un módulo más general de teoría de la mente.
\end{abstract}

Palabras clave: pragmática, modularidad, modularidad masiva, arquitectura mental, ciencias cognitivas 


\begin{abstract}
One of the fundamental problems with the cognitive nature of pragmatics concerns its place in the architecture of the mind. In this regard there are several proposals. One holds that part of the central systems within the framework of the theory of modular Jerry Fodor. However, Asa Kasher raises a hypothesis based on Fodor modulates architecture, but it gives a character to the modular least some aspects of pragmatic interpretation. In this work we argue that both proposals are incorrect due to inconsistencies with the empirical findings on pragmatic processing. Rather, we support a hypothesis based on the massive modularity of mind, where evolutionary considerations led to the emergence of a pragmatic sub-module that is part of a more general theory of mind module.
\end{abstract}

Keywords: pragmatics, modularity, massive modularity, menta architecture, cognitive sciences 


\section{Introducción}

En sus inicios la pragmática nació como una rama de la filosofía del lenguaje. Sin embargo, el desarrollo de este campo prontamente se movió hacia la interdisciplinariedad. Una de las teorías más importantes de la pragmática, la teoría de la relevancia (Sperber y Wilson, 2004; 1995), estudia cómo se construyen inferencias dentro de un procesamiento mental que es responsable de la interpretación de la comunicación humana. Con ello, la pragmática se movió hacia los estudios de la ciencia cognitiva. El resultado es un marco explicativo el cual está incluido en los estudios amplios de la cognición humana.

De acuerdo con este giro, la presente investigación considera a la pragmática de índole cognitiva como el estudio de los estados mentales de las personas que participan en un evento comunicativo (Bara, 2010). El análisis de las interacciones comunicativas y sus estados mentales subyacentes implican determinar las motivaciones individuales, creencias, objetivos, deseos e intenciones y, especialmente cómo estos estados mentales se relacionan con el componente lingüístico de la comunicación. A su vez, los estados mentales y las elecciones lingüísticas que se emplean en un evento comunicativo están determinadas en distinto grado por las condiciones contextuales en que dichos eventos ocurren. Esta concepción delimita una serie de fenómenos comunicativos que caen dentro el dominio de la pragmática. Entre estos fenómenos podemos encontrar los actos de habla, las presuposiciones, las implicaturas, las expresiones deícticas, las reglas conversacionales, el lenguaje no literal, reglas de cortesía, etc. (Airenti, 2017).

Bajo este marco, uno de los temas que corresponde determinar es la descripción de la arquitectura cognitiva (Thagard, 2012) y el rol funcional de la pragmática al interior de dicha arquitectura (Carston y Powell, 2008; Fodor, 2000; Segal, 1996; Sperber, 2002; Sperber y Wilson, 2002; Wilson, 2005). Esto implica evaluar qué tipo de sistema es responsable del procesamiento pragmático. ¿Es un sistema de resolución de problemas de carácter general o dedicado? ¿Con qué otro tipo de sistema interactúa? La presente investigación tiene como objetivo evaluar diversas teorías respecto del lugar del procesamiento pragmático en la arquitectura mental. En este sentido, proponemos que una teoría respecto del lugar del procesamiento pragmático en la arquitectura mental debe ser coherente con consideraciones propias de la psicología evolutiva concerniente al tipo de sistema cognitivo que ha sido naturalmente seleccionado para resolver un problema adaptativo particular (Sperber, 2002). Ello conlleva preguntarse ¿responde el procesamiento pragmático a un problema adaptativo que ha llevado a que emerja un sistema cognitivo que se encargue de dichos problemas?, o en su defecto ¿̇basta un mecanismo de orden general para explicar el procesamiento pragmático? 
Probablemente la posición más influyente en torno a la arquitectura cognitiva se atribuye a Jerry Fodor (1983; 2000). Desde su perspectiva la mente posee una arquitectura híbrida. Ella se compone, por un lado, de sistemas perceptuales de input, incluyendo la percepción del lenguaje; sistemas de outputs y por otro, de un sistema central. Mientras que los sistemas de input y outputs son módulos mentales autónomos, el sistema central no es modular y es responsable de la formación de creencias y de la toma de decisiones, entre otras conductas. Para Fodor (2000), los procesos de inferencia pragmática son una función del sistema central: su objetivo es fijar una creencia respecto del significado del hablante (el contenido de su intención comunicativa) y dicho proceso es altamente sensible al contexto. Por lo tanto, el autor concluye que la fase de decodificación lingüística de un enunciado puede ser realizada por un sistema modular, mientras que la fase inferencial, la cual vincula la brecha entre el significado lingüístico y el significado pretendido por el hablante, es no modular. Sin embargo, también existen posiciones, dentro del marco de la teoría de la arquitectura mental de Fodor, que sostienen que la dimensión pragmática del lenguaje tiene un componente modular (Kasher, 1991). Bajo esta teoría, ciertos fenómenos pragmáticos se procesan al interior de un módulo pragmático lingüístico, mientras que otros ocurren en el sistema central. Desde nuestra perspectiva se considera que ambas propuestas poseen problemas que impiden explicar idóneamente, dentro del contexto de la psicología evolutiva, cómo ocurre el procesamiento pragmático. Por el contrario, nuestro objetivo es apoyar la idea de que la pragmática sí constituye un módulo especializado, pero dentro de un marco de una propuesta distinta al marco fodoriano. A saber, la teoría de la modularidad masiva de la mente (Carruthers, 2006; Sperber, 2002). En esta teoría, la mente se compone exclusivamente de módulos especializados dentro de los cuales uno de ellos estaría dedicado al componente pragmático del lenguaje (Sperber, 2000; Sperber y Wilson, 2002; Wilson, 2000; 2005).

En la primera sección se expondrán los principales postulados de la teoría modular de la mente de Jerry Fodor $(1983 ; 2000)$. Se pondrá énfasis en las características que poseen los módulos de la mente dentro de esta teoría. Finalizaremos esta sección con el lugar que otorga dicho autor a la pragmática en el marco de su teoría modular. En el segundo apartado revisaremos críticamente la teoría modular pragmática de Asa Kasher (1991). Dicho autor asume la teoría fodoriana de la mente, pero se diferencia de Fodor en el sentido en que sí existe un módulo pragmático lingüístico encargado de al menos, algunos fenómenos pragmáticos (la fuerza de los actos de habla). A continuación, presentaremos una serie de objeciones a la propuesta de Kasher que nos llevarán a concluir que su propuesta es poco plausible.

En el tercer apartado presentamos las bases para la propuesta respecto del problema que guía la presente investigación. Nuestra propuesta afirma la existencia de un módulo 
pragmático, pero no dentro del marco de una teoría modular fodoriana, sino dentro de la teoría de la modularidad masiva (Carruthers, 2006; Sperber 2002; Wilson, 2005). Para ello, en la tercera sección se presentarán los principales aspectos de dicha teoría, enfatizando el concepto amplio de módulo que utilizan diferenciándolo con la propuesta de Fodor (2000). En el cuarto apartado presentamos argumentos en favor de la existencia de un submódulo pragmático que forma parte de un módulo más amplio responsable de la atribución de estados mentales (habilidad frecuentemente denominada Teoría de la Mente). Dicho submódulo pragmático se apoya en un par de principios que regulan la comunicación (Sperber y Wilson, 1995; 2004) y de procedimientos de procesamiento exclusivos para la detección de los significados intencionales de índole lingüística y también ostensiva. Finalmente, concluimos con una serie de proyecciones y asuntos que debe asumir una teoría pragmática modular en el contexto de la teoría modular masiva.

\section{La teoría modular de la mente y el procesamiento pragmático}

Una condición general que toda teoría del funcionamiento de la mente debe explicar es cómo la mente construye representaciones de estados de cosas que son externas a ella (Stillings, y otros, 1995; Von Eckardt, 2012). Fodor (1980) sostiene que las representaciones consisten en estructuras semejantes a las estructuras lingüísticas (language-like structures) dentro de un lenguaje interno del pensamiento. Sin embargo, estas consideraciones generan otro tipo de preguntas que es necesario abordar. Una de ellas se refiere a cuál es la naturaleza de la relación entre esas representaciones. Según Fodor (1980), esas relaciones son expresadas en términos de propiedades formales y sintácticas que se separan de las propiedades semánticas. Esta distinción entre los atributos semánticos de las representaciones y las propiedades sintácticas internas es integral a las teorías de Fodor (1980; 1983; 2000) y a las de Chomsky (1980).

Fodor explica de qué forma esas representaciones son manipuladas para realizar las variadas operaciones cognitivas de nuestra mente. Para ejecutar esas operaciones, como por ejemplo las inferencias o el procesamiento lingüístico, la mente debe ser capaz de manipular las representaciones a las que accede. La manipulación procede por medio de propiedades sintácticas y formales de las representaciones de un modo semejante a las computaciones que ejecuta un computador (Fodor, 1980).

El contenido semántico de las representaciones no es esencial a las operaciones de manipulación de dichas representaciones. Consideremos el siguiente ejemplo. Supongamos que nuestra mente tiene acceso a dos representaciones $\mathrm{P}$ y $\mathrm{Q}$, donde $\mathrm{P}$ corresponde a la proposición 'Héctor está feliz' y Q corresponde a la representación 'Si Héctor está Feliz entonces irá a bailar'. La conclusión basada en la regla modus ponens es R ('Héctor irá a bailar'). Para llegar a $\mathrm{R}$ es necesario realizar una computación que procede desde las propiedades 
formales de $\mathrm{P}$ y Q y, específicamente, a partir de la identidad formal de $\mathrm{P}$ con el antecedente de Q. Que 'Héctor' refiere a un hombre particular y que "irá a bailar” refiere a la discoteca de la calle principal son representaciones semánticas de P y Q y, como tal, no son parte de la manipulación formal de las representaciones.

La teoría modular propone que la mente posee una compleja organización estructural. A nivel global, la mente posee distintas facultades innatas interrelacionadas. Por ejemplo, Chomsky (1966) sostiene que existe una facultad innata del lenguaje en nuestras mentes. Esta facultad involucra manejar los aspectos computacionales del lenguaje. Estos aspectos incluyen las reglas fonológicas, semánticas, pero principalmente las reglas sintácticas de carácter universal.

A nivel local, la mente se organiza en torno una serie de "módulos cognitivos". Revisemos a continuación cada una de las características que poseen los módulos según Fodor (1983).

- Los módulos son de dominio específico: Cada módulo está restringido al tipo de contenido que puede tomar como input. Solo puede procesar aquellos contenidos que constituyen su dominio. Así, el sistema visual está restringido a los inputs visuales; el sistema auditivo a los inputs auditivos, etc.

- Los módulos generan outputs simples o superficiales (no conceptuales): el output de un módulo es superficial en el sentido en que no es conceptual. Por lo tanto, lo módulos generan información de varios tipos, pero ellos no generan creencias o estados mentales. Por el contrario, según Fodor (2000), la fijación de creencias es un arquetipo de sistemas no modulares. Por ejemplo, el módulo visual podría entregar una representación de la superficie y los contornos de algún objeto del campo visual, pero no podría identificar dicho objeto con una silla, ni mucho menos generar la creencia de que la silla está presente.

- Los módulos son innatos y localizados: El desarrollo de los módulos y su crecimiento se encuentra bajo el control genético. Además, ellos se encuentran localizados en estructuras específicas del cerebro.

- Los módulos son obligatorios y rápidos en su procesamiento: Sus operaciones no están bajo el control voluntario, y ellos generan outputs más rápido que el sistema central no modular.

- Los módulos están encapsulados y son inaccesibles: Ellos se encuentran aislados de los demás módulos de la cognición y poseen operaciones internas que son inaccesibles a otros módulos. Estas dos propiedades están vincu- 
ladas a la especificidad de dominio, pero son distintas de ella. Decir que un sistema de procesamiento está encapsulado es decir que sus operaciones internas no pueden generarse en virtud de información contenida fuera de dicho módulo. En contraste, sostener que un sistema es inaccesible significa que los demás módulos no poseen acceso a su procesamiento interno, sino que solo a sus outputs. Por otra parte, la especificidad de dominio se refiere a las restricciones sobre el input de un sistema. Afirmar que un sistema es específico de dominio significa que puede procesar solo inputs de un tipo particular, mientras que las propiedades de la encapsulación e inaccesibilidad corresponden a relaciones de acceso que se obtienen entre las operaciones internas de esos sistemas y los demás módulos. Por lo tanto, es posible que un módulo pueda carecer de especificidad de dominio y a la vez estar encapsulado y ser inaccesible (Carruthers, 2006; Sperber, 2002).

Los módulos cognitivos proveen los inputs a un sistema central el cual, a diferencia de los módulos, no son específicos de ningún dominio y tampoco están informacionalmente encapsulados. Este sistema central es el lugar donde ocurren las inferencias y, como tal, está asociado a procesos de formación de creencias y resolución de problemas.

¿Qué lugar tiene la pragmática en esta propuesta arquitectónica de la mente? De acuerdo con la perspectiva fodoriana clásica, la pragmática y las habilidades de lectura de intenciones (mind-reading) son sistemas cognitivos centrales los cuales son utilizados para atribuir estados mentales a los demás sobre la base de habilidades de razonamiento de propósito general. Fodor $(1983 ; 2000)$ combinó la perspectiva chosmkiana del lenguaje (Chomsky, $1966 ; 1980)$ con la perspectiva griceana de la pragmática (Grice, 1975; 1989). En su teoría, el lenguaje es un sistema modular autónomo y de dominio específico y el procesamiento pragmático es parte del sistema central de dominio general. La diferencia entre ambos sistemas no es el hecho de que la pragmática sea de carácter inferencial y el lenguaje no, sino que las inferencias pragmáticas son fuertemente dependientes del contexto y no existe en principio ningún límite respecto del tipo de información contextual que podría requerirse ni tampoco la fuente de dicha información. Para Fodor (2000), la interpretación pragmática es un proceso central debido a que su resultado depende de factores "globales" tales como el acceso libre a información de la memoria a largo plazo. Los procesos modulares, en contraste, son 'locales', 'encapsulados' y fuertemente restringidos al tipo de fuente de información en la cual ellos operan. De esta manera, el procesamiento pragmático en la arquitectura mental de Fodor no constituye un módulo específico de la mente, sino que es parte del aparato inferencial del sistema central multipropósito. 


\section{La pragmática modular de Kasher}

Kasher (1991) asume que la modularidad es una descripción correcta de nuestras capacidades mentales. Su objetivo, sin embargo, es sostener que sí existe un componente modular de la pragmática. Para ello, Kasher propone la siguiente fórmula básica de la arquitectura mental de la pragmática:

Arquitectura mental de la pragmática $=$ un módulo pragmático + una parte pragmática del procesador central + una interfaz pragmática.

Para llegar a esta descripción tripartita, Kasher examinó seis fenómenos pragmáticos: deixis (Cummings, 2005), presuposiciones léxico-pragmáticas (Cummings, 2005), fuerza de los actos de habla (Searle, 1975), actos realizativos (Austin, 1998), implicaturas conversacionales (Grice, 1975) y principio de cortesía (Leech, 1983). El autor argumenta que el procesamiento de los actos de habla requiere de un módulo propiamente pragmático que es lingüístico en su naturaleza; las implicaturas conversacionales y el principio de cortesía sugieren la presencia de un componente pragmático en el procesador central de la mente y los elementos deícticos junto a las presuposiciones pragmáticas proveen evidencia respecto de la existencia de una interfaz entre el módulo pragmático y el procesador central. A continuación, se presenta cada uno de los componentes de la arquitectura mental de la pragmática según Kasher (1991).

- Módulo pragmático

Kasher (1991) sostiene que el procesamiento de los actos de habla y, particularmente, los actos de habla indirectos requieren de la existencia de un módulo pragmático lingüístico. Este módulo es responsable del procesamiento sintáctico y semántico de los enunciados, desde los cuales se obtiene el componente o fuerza literal del acto de habla. En este sentido, la construcción de la dimensión locutiva involucra la atribución de un estado intencional basado en esa fuerza literal. Sin embargo, para explicar que la fuerza pretendida en diversos actos de habla difiere de la fuerza que es codificada en la expresión lingüística -actos de habla indirectos-, la fuerza literal del enunciado opera como presuposición al interior del procesador central. En este sistema, dicha presuposición -derivada de la decodificación lingüística- interactúa con las creencias sobre las intenciones comunicativas del hablante, con el resultado de que algunas presuposiciones se mantienen -en el caso de que la fuerza literal de una expresión sea tomada como la fuerza pretendida por el hablante-, otras presuposiciones son rechazadas, e incluso otras presuposiciones son utilizadas y luego rechazadas. En 
este sentido, el componente modular pragmático propuesto por Kasher (1991) es el encargado de construir la presuposición de estado intencional que se deriva de la interpretación literal de un enunciado.

\section{- Sistema central}

Kasher apela a un sistema central para explicar el componente pragmático del lenguaje referente a las implicaturas conversacionales (Grice, 1975, 1989) y los principios de cortesía (Leech, 1983). Para ello, se basa en un argumento fundamental de la teoría de las implicaturas de Grice (1975). Nos referimos a que el principio de cooperación y las máximas están basados en un principio general de acción racional.

Aplicado al dominio de la acción, la racionalidad se basa en un principio de medios efectivos. De este modo, un agente racional elige aquellas acciones que son más efectivas de acuerdo con sus objetivos y con un mínimo costo. Es imposible circunscribir el rango de factores que juegan un papel en la elección de tales acciones. Por esta razón, Kasher sitúa el principio general de racionalidad al interior del sistema central.

Obviously, our principles of general rationality and of effective means are not domain specific in any reasonable sense and hence, if they constitute the principles of operation of some mental device, then the latter must be a central cognitive system which employs the former principle in forming beliefs and in planning action, not only when language plays an overt role. (Kasher 1991:578)

Por lo tanto, según Kasher (1991), si las implicaturas conversacionales están regidas por un principio que se sitúa al interior de los sistemas centrales, entonces el procesamiento de las implicaturas también ocurre en dichos sistemas.

Con respecto a la cortesía, Kasher sostiene que la aplicación apropiada del principio de cortesía demanda el conocimiento de una serie de factores al interior del contexto de un enunciado. Siguiendo a Leech (1983), el autor sostiene que estos principios varían según aspectos culturales y sociales. Tal dependencia contextual implica la localización de estas operaciones en un sistema central.

- Interface entre módulo pragmático y sistema central

De acuerdo con Kasher (1991), existen dos fenómenos pragmáticos que sugieren la existencia de interfaces entre un módulo específico y un procesador central. Las expresiones deícticas, tales como 'él' o 'aquí' revelan un complejo grupo de características de interface entre el lenguaje y módulos perceptivos por un lado, y entre el sistema central por el otro. En este 
sentido, el referente de 'ella', en un enunciado, no está determinado solo por operaciones de un módulo lingüístico. Incluso si tal módulo existiese y contuviera el conocimiento de que 'ella' refiere a un ser humano femenino, la identificación del referente de 'ella', según Kasher, depende de la operación de un dispositivo central el cual posee acceso a información del módulo perceptual.

\subsection{Problemas de la teoría de Kasher}

En la presente investigación, consideramos que la propuesta de Kasher genera una serie de problemas que la hacen insostenible como propuesta respecto de la arquitectura mental de la pragmática. Específicamente, para conceder la plausibilidad de la hipótesis respecto de que la fuerza de los actos de habla se procesa en un módulo pragmático lingüístico, primero se debe estar seguro de que el tipo de creencias que se identifica con la operación del sistema central no son esenciales al procesamiento que ocurre en el módulo pragmático-lingüístico. Sin embargo, si se da el caso de que existan procedimientos del sistema central que influyen en la decodificación de la fuerza literal de un acto de habla, entonces la hipótesis de un módulo pragmático pierde plausibilidad, pues viola las propiedades de especificidad de dominio, aislamiento y encapsulamiento. Supongamos el siguiente ejemplo:

¿Cómo se dice, hijo; cuando te dan una carta?'

Este enunciado tiene la fuerza literal de una pregunta. Sin embargo, también puede interpretarse como una petición (el hablante quiere que su hijo le agradezca por algo que recientemente ha recibido). De acuerdo con Kasher (1991), la fuerza literal de este enunciado entra al sistema central como una presuposición. En este sistema interactúa con una serie de creencias basadas en el conocimiento del contexto en el cual ocurre el enunciado. Mediante la interacción entre la presuposición y el conjunto de creencias del sistema central, se rechaza la fuerza literal y se la reemplaza por la fuerza de una petición. Sin embargo, contrario a lo que sostiene Kasher, existen casos en que las creencias del sistema central pueden determinar la asignación de la fuerza literal de un enunciado. Consideremos la palabra 'carta'. Podemos asignar tres posibles significados de la palabra: objeto de juego de mesa, baraja, correspondencia escrita y menú de restaurante. Para llegar al contenido proposicional explícito del enunciado se debe escoger solo uno de los significados propuestos de la palabra 'carta'. Claramente, la información visual que se obtiene del contexto físico de la expresión es por sí misma suficiente para lograr la desambiguación de la palabra. Sin embargo, de acuerdo con las suposiciones de una teoría modular, la información visual es procesada en primera instancia por un módulo perceptual visual y solo después de este procesamiento se vuelve accesible la información a una serie de procesos cognitivos centrales para formar la creencia de dicha percepción. La desambiguación sería uno de estos procesos. Se despren- 
de de esto que el módulo pragmático sí es permeable a la información que es manipulada en el procesador central. Esto viola el principio de encapsulamiento informacional de cada módulo. Por lo tanto, no existe un módulo pragmático-lingüístico de carácter discreto.

Por otro lado, la presunción de que el principio de racionalidad ocurra solo al interior de un sistema central parece poco probable, puesto que el principio de racionalidad que subyace a la comunicación humana no es exclusivo de dicha facultad. Cummings (2005) sugiere que el principio de racionalidad de Grice (1989) no se circunscribe solamente a la acción intencional de los seres humanos, sino que es un principio que guía todas las operaciones biológicas. Es decir, las operaciones de un módulo, por ejemplo, visual perceptual, también están guiados por el principio de la adquisición de mayor beneficio informacional con menor costo de procesamiento. Por lo tanto, la idea de que las implicaturas se sitúan en el procesador central debido a que ellas se derivan de un principio general de racionalidad es imprecisa, pues incluso los módulos responden a este principio.

\section{Modularidad masiva}

Una de las críticas fundamentales a la arquitectura modular de la mente provienen desde el enfoque de la cognición corpórea (Barsalou, 2010). De acuerdo con diversas investigaciones (Desai, Binder, Conant y Seidenberg, 2010; Fischer y Zwaan, 2008), existe evidencia de que durante el procesamiento del lenguaje ocurre una coactivación de zonas cerebrales asociadas a la percepción y a aspectos motrices, incluso en etapas preconceptuales (Ostarek y Huettig, 2017). En este sentido, las propiedades de los módulos fodorianos han sido criticadas, especialmente aquellas referidas a la encapsulación. Si la construcción de significado durante el procesamiento del lenguaje depende de la coactivación de zonas perceptuales y motrices, entonces la idea de que los módulos son inaccesibles y están asilados de las operaciones de otros módulos no sería sostenible bajo un marco explicativo de la cognición corpórea.

Estos cuestionamientos llevaron a diversos científicos a proponer modelos alternativos en torno a la arquitectura cognitiva. Uno de ellos corresponde a la teoría de la modularidad masiva de la mente (Carruthers, 2006). En esta perspectiva se asume que la mente funciona exclusivamente en virtud de distintos módulos sin la necesidad de apelar a sistemas centrales. Sin embargo, la noción de módulo utilizada en esta teoría se basa en criterios diferentes a los aplicados por Fodor $(1983,2000)$. Bajo la teoría de la modularidad masiva, la arquitectura de la mente responde al resultado de consideraciones evolutivas (Carruthers, 2006; Sperber, 2002). El supuesto que se sigue es que la selección natural favorece soluciones específicas a problemas específicos. Un procedimiento cognitivo dedicado a tratar con un problema ambiental particular y recurrente es muy probable que sea más eficiente que un procedimiento más general 
aplicado al mismo problema. De esta forma, la perspectiva evolucionista (Barkow, Cosmides y Tooby, 1992) sugiere el incremento y refinamiento de la modularidad mental en vez de cualquier tipo de fusión entre sistemas más generales para tratar con los problemas contingentes de un organismo. La propiedad crucial de un sistema modular, bajo esta perspectiva, corresponde a que, a la hora de enfrentar estos problemas ambientales, un mecanismo de propósitos especiales, sintonizado con las regularidades del dominio particular del problema, será más eficiente que un mecanismo de propósitos generales.

Sin embargo, la tesis de la modularidad masiva no puede utilizar el mismo concepto de módulo que utiliza Fodor (1983). Algunas propiedades de los módulos de Fodor necesitan abandonarse en la medida en que se asume que no existen sistemas centrales. En la teoría de la modularidad masiva existen módulos que pueden perfectamente generar outputs puramente conceptuales (por ejemplo creencias). Por lo tanto, la propiedad de superficialidad de los outputs no se aplica a los módulos en el marco de la teoría de la modularidad masiva.

La especificidad de dominio también debe abandonarse o reconceptualizarse en términos funcionales antes que en términos de dominios de contenidos o inputs específicos. Recordemos que, por especificidad de dominio, Fodor (1983) sugiere que los módulos pueden procesar solo un tipo de inputs, aquellos que pertenecen a su dominio. Sin embargo, los psicólogos evolucionistas que están a favor de la teoría de la modularidad masiva entienden la especificidad de dominio de una manera bastante diferente. En la perspectiva evolucionista el dominio de un módulo es lo que se supone que realiza, en vez del tipo de contenido que puede recibir como input (Carruthers, 2006; Sperber, 2002; Wilson, 2005). En la teoría de la modularidad masiva pueden existir módulos que reciban inputs de distinta naturaleza. Un ejemplo de ello es el módulo de la Teoría de la Mente (Wilson, 2005). Este módulo recibe inputs tanto de información visual, verbal o de otro tipo.

¿Cuál es el objetivo detrás de la reconfiguración de la arquitectura fodoriana? La teoría de la modularidad masiva está basada en consideraciones extraídas de la psicología evolutiva (Barkow, Cosmides, y Tooby, 1992). Una propuesta de arquitectura mental debe considerar el desarrollo evolutivo íntegramente. Es decir, la organización de la mente debe responder a las presiones de selección natural y adaptación. La propuesta de Fodor no considera dichos aspectos y si bien posee coherencia interna, carece de plausibilidad biológica en el sentido evolutivo (Carruthers, 2006).

\section{La teoría pragmática modular de Sperber y Wilson}

Si se asume la perspectiva de la modularidad masiva, la pregunta concerniente a la pragmática es si ella forma parte de un módulo lingüístico, de un módulo general de Teoría de la Mente o de algún otro módulo específico. Una de las posiciones al respecto es que la 
atribución del significado del hablante cae dentro del dominio de la capacidad de Teoría de la Mente, la cual consiste en la atribución de intenciones, creencias y otros estados mentales sobre la base de cualquier tipo de propósitos conductuales, sea este comunicativo o no comunicativo. Es decir, la interpretación pragmática ocurre en el módulo de la Teoría de la Mente (Bloom, 2002). Sin embargo, para Sperber y Wilson (Sperber, 2000; Sperber y Wilson, 2002; Wilson, 2000; Wilson, 2005), los procesos pragmáticos son realizados por un módulo de comprensión dedicado, de dominio específico y con sus propios principios y procedimientos. Ello porque existen regularidades manifiestas de las conductas comunicativas ostensivas (de las cuales la conducta verbal es la principal) que las diferencian de las conductas no comunicativas. De esta forma, estas regularidades generaron filogenéticamente el surgimiento de un módulo dedicado exclusivamente a la comprensión pragmática mediante la atribución de intenciones. Esto debido a que existen diferencias entre la atribución de intenciones en términos genéricos y la atribución de intenciones mediante la conducta lingüística.

Desde una perspectiva evolucionista (Wilson, 2005), lo que caracteriza a un módulo es la presencia de mecanismos dedicados (típicamente adaptaciones biológicas a regularidades en algún dominio) los cuales no pueden ser vistos como casos especiales de mecanismos más generales operando en un dominio amplio (Sperber, 2002). Para ejemplificar, consideremos las habilidades de Teoría de la Mente, las cuales permiten a los humanos predecir y explicar la conducta de otros en términos de sus estados mentales -creencias, deseos, intenciones, etc.- (Baron-Cohen, 1997; Carruthers y Smith, 1996). De acuerdo con la versión clásica de la tesis modularista (Fodor, 1983, 2000), la Teoría de la Mente debiese ser un proceso central antes que uno modular, porque su resultado depende de factores globales: no existe en principio una restricción en el tipo de información contextual que se requiere para predecir y explicar la acciones de los demás. Desde en una perspectiva evolucionista (Wilson, 2005), la cuestión no se refiere tanto a si los procesos involucrados son globales o locales, sino a si son realizados por mecanismos de propósito general o por mecanismos autónomos de propósitos especiales en sintonía con las regularidades existentes solo en el dominio de la conducta intencional. En la medida en que la Teoría de la Mente involucre tales mecanismos inferenciales de propósitos específicos, sería de carácter modular, pero en un sentido amplio.

De acuerdo con Grice $(1975,1989)$, la interpretación de un enunciado es un ejercicio de Teoría de la Mente. La interpretación del significado del hablante implica el reconocimiento de la intención comunicativa detrás de la conducta lingüística. Esto constituye un caso especial de un problema más general consistente en explicar la conducta de un individuo en términos de la atribución de estados mentales. 
Tradicionalmente se ha descrito la teoría de la mente como un ejercicio de razonamiento reflexivo (un proceso central en términos de Fodor). Este ejercicio involucra la aplicación de habilidades de razonamiento general basadas en premisas explícitas e implícitas sobre las relaciones entre los estados mentales y la conducta. La propuesta de Grice (1975) es coherente con esta aproximación. Por ejemplo, su esquema para la derivación de las implicaturas conversacionales es un ejercicio estándar de teoría de la mente:

He said that P; he could not have done this unless he thought that Q; he knows (and knows that I know that he knows) that I will realize that it is necessary to suppose that Q; he has done nothing to stop me thinking that Q; so, he intends me to think, or is at least willing for me to think, that Q. (Grice, 1975:30-31)

Un oyente que siga la ruta de Grice tendría que incurrir en un complejo proceso de razonamiento discursivo para identificar una sola implicatura. Sin embargo, existe evidencia que sugiere una perspectiva más modular (Cummings, 2009). La mayoría de esta evidencia proviene de las pruebas que miden la Teoría de la Mente tales como las tareas de falsas creencias, en las cuales los participantes deben rastrear las creencias de los otros (Baron-Cohen, 1997). Tal habilidad puede variar en distintos grados. Los niños generalmente pasan las pruebas de creencias de primer orden a la edad de cuatro años, mientras que las personas con Trastorno del Espectro Autista (TEA) típicamente tienen problemas para reconocer intenciones (Happé, 1993; Baron-Cohen, 1997).

Las investigaciones en torno a la comunicación inferencial y la Teoría de la Menten sugieren una disociación entre ambos fenómenos. Estas disociaciones no son explicadas por la perspectiva griceana y fodoriana tradicional de Teoría de la Mente como un ejercicio de razonamiento de propósito general. Por ejemplo, el Síndrome de Williams (SW) es un extraño desorden genético que resulta en un bajo promedio de coeficiente intelectual (CI), combinado con buenas habilidades lingüísticas y sociales (Cummings, 2009). Sin embargo, dichos sujetos poseen pobres habilidades de razonamiento general. Ellos pasan las tareas de Teoría de la Mente con facilidad, mientras que sus habilidades para razonar en términos generales están seriamente dañadas (Segal, 1996). Esto sugiere que la teoría de la mente no puede ser un proceso consciente y reflexivo como el tipo de procesos ilustrado por Grice, sino que depende de un mecanismo inferencial dedicado el cual podría permanecer intacto al mismo tiempo que otros mecanismos de razonamiento de propósitos generales podrían estar dañados. Estas disociaciones también pueden ocurrir en la dirección opuesta. Por ejemplo, las personas con TEA grado 1 pueden tener buenas habilidades de razonamiento general combinadas con serias dificultades en habilidades de Teoría de la Mente (Cummings, 2009). Por lo tanto, la evidencia neuropsicológica y de estudios del desarrollo 
parece favorecer una perspectiva de la teoría de la mente como un sistema modular de dominio específico antes que un proceso central reflexivo.

Todos estos hallazgos llevaron a Sperber y Wilson (2002) a sostener que la Teoría de la Mente no es un sistema central en el sentido fodoriano, sino que un módulo inferencial de dominio específico (o un grupo de módulos). Además, Wilson (2005) aplica una perspectiva modular masiva de la mente a la relación entre pragmática y Teoría de la Mente. Del mismo modo en que existen razones para pensar que la Teoría de la Mente no es meramente una aplicación de habilidades de razonamiento general a un dominio particular (dominio conductual), también existen buenas razones para pensar que la interpretación pragmática no es meramente la aplicación de habilidades de Teoría de la Mente a un dominio particular lingüístico o comunicativo ostensivo. La comunicación verbal presenta dificultades especiales y exhibe ciertas regularidades que no se encuentran en otros dominios. Este tipo de regularidades podría conllevar el desarrollo de un módulo de comprensión dedicado a la comunicación verbal y ostensiva, con sus propios principios y mecanismos (Sperber, 2000; Sperber y Wilson, 2002; Wilson, 2000; Wilson, 2005). Incluso, propuestas recientes han sugerido la existencia de un mecanismo adicional cuya función es determinar la confiabilidad de los hablantes y sus enunciados. Este mecanismo de vigilancia epistémica sería de carácter específico entendido en términos de la teoría de la modularidad masiva (Mazzarella, 2016; Mazzarella y Pouscoulous, 2021).

Según lo mencionado anteriormente, la perspectiva psicológica evolutiva trata a la cognición como una función biológica y a los mecanismos cognitivos como adaptaciones (Barkow, Cosmides y Tooby, 1992). La mente es un grupo de mecanismos cognitivos dedicados, los cuales evolucionaron mediante pequeños pasos incrementales, típicamente involucrando la selección de mecanismos como variantes que realizan más eficientemente (generando mayores beneficios con menores costos) que otras variantes de mecanismos (Sperber, 2002; Carruthers, 2006). Una forma en la que un mecanismo cognitivo dedicado puede contribuir a la eficiencia de una tarea es proveyendo procedimientos inferenciales de propósitos especiales en sintonía con las regularidades de algún dominio particular, el cual genera conclusiones confiables solo cuando se aplica al input de dicho dominio. Por ejemplo, la perspectiva modular de la Teoría de la Mente apela a procedimientos inferenciales de propósito específico los cuales trabajan confiablemente en el dominio de la conducta intencional, produciendo las mismas conclusiones derivables mediante mecanismos de razonamiento de propósito general, pero de una forma más eficiente (en relación con los parámetros de costo-beneficio). En estos mecanismos más rápidos y sencillos, las regularidades en las relaciones entre los estados mentales y la conducta no necesitan estar representadas y utilizadas como premisas explícitas en un proceso inferencial. Por lo tanto, 
la propuesta de Grice (1975) respecto del reconocimiento de intenciones como una inferencia de propósito general se simplifica mediante la actividad de un módulo que recoge las regularidades propias del dominio de la conducta intencional. El resultado debiese ser una habilidad más automática e intuitiva respecto de cómo extraer conclusiones válidas en el dominio de la conducta intencional, pero sin ningún tipo de consciencia reflexiva respecto de cómo esas conclusiones son construidas.

Un ejemplo de un submódulo de teoría de la mente investigado corresponde a la Detección de la Dirección de la Mirada (Eye Direction Detector), la cual es vista como una habilidad presente tempranamente en los infantes para inferir lo que las personas están observando a partir de la dirección de su mirada (Baron-Cohen, 1997; Tomasello, 2003). Un infante que realiza este tipo de inferencias no está utilizando un módulo de Teoría de la Mente general e internamente no diferenciado para derivar conclusiones como las de (3) sobre la base de premisas explícitas de (1) y (2):

(1) Si una persona P fija sus ojos en dirección al objeto $\mathrm{O}$, entonces $\mathrm{P}$ está observando $\mathrm{O}$.

(2) Papá está fijando sus ojos en dirección al gato.

(3) Papá está observando el gato.

Parece más razonable asumir que los seres humanos están equipados desde la infancia con un Detector de Dirección de la Mirada, el cual explota la fuerte correlación entre la dirección de la mirada y la percepción visual y directamente atribuye estados perceptuales y atencionales en virtud de la dirección de la mirada (moviendo directamente desde las premisas tales como (2) a conclusiones tales como (3)). Esta atribución puede en sí misma proveer un input para otros dispositivos dedicados, tales como los involucrados en la adquisición de palabras (Tomasello, 2003).

A pesar del hecho de que las habilidades de la Teoría de la Mente pueden ser descompuestas en una serie de módulos subespecializados, la perspectiva estándar ha sostenido que la atribución de estados mentales requerida para la comprensión pragmática será automáticamente generada por mecanismos de Teoría de la Mente generales los cuales aplican a todos los dominios. Sin embargo, Sperber y Wilson (Sperber, 2000; Sperber y Wilson, 2002; Wilson, 2000; Wilson, 2005) sostienen que existen serios problemas en esta perspectiva. En primer lugar, el rango de intenciones que puede ser razonablemente atribuido a un agente sobre la base de alguna conducta no comunicativa en una situación particular es generalmente bastante limitada (por ejemplo, si extiendo mi brazo hacia la alacena será para sacar una tasa o un vaso). Sin embargo, en la comunicación lingüística el entorno físico coloca 
pocas restricciones al contenido de un enunciado (comparado con el caso anterior). Esto genera que exista una brecha entre el significado lingüístico y el significado pretendido por el hablante. Es decir, existe un vasto rango de significados posibles que en un momento dado un hablante pudo o quiso comunicar. No queda claro cómo los procedimientos estándar para atribuir las intenciones sobre la base de la conducta no comunicativa puedan generar la atribución adecuada del significado del hablante, excepto en casos triviales (Sperber, 2002; Sperber y Wilson, 2002).

En segundo lugar, mientras ambos tipos de atribución de estados mentales involucran claramente capacidades metarepresentacionales (la capacidad de representar las representaciones mentales de los otros), existe una importante diferencia en la complejidad de la aplicación de ambos casos. La comunicación ostensiva involucra tanto una intención informativa como una intención comunicativa (Sperber y Wilson, 2004; 1995), de tal forma que el oyente debe reconocer que el hablante intenta hacerle creer que él pretende que su oyente crea un cierto grupo de proposiciones (Sperber, 2000). En este caso existen 3 niveles de metarepresentación, mientras que en la comprensión de acciones ordinarias no comunicativas solo es necesario un único nivel de atribución de intención. Por lo tanto, existen dos procedimientos distintos de atribución de intenciones. Uno es activado por la comunicación lingüística (o cualquier comunicación ostensiva) y otra por la observación de la conducta intencional no comunicativa. En este sentido, para la teoría de la relevancia, la pragmática es un submódulo de un módulo de la Teoría de la Mente que utiliza sus propios principios y procesos. A continuación, describiremos cuáles son dichos principios y regularidades.

\subsection{Pragmática como un submódulo del módulo de teoría de la mente}

La teoría de la relevancia (Sperber y Wilson, 2004; 1995) es una teoría sobre la cognición y la comunicación humana basada en la definición de la relevancia y dos principios generales: un primer principio cognitivo de relevancia y un segundo principio comunicativo de relevancia. La relevancia es caracterizada como una propiedad de los inputs de un proceso cognitivo y analizado en término de las nociones de efecto cognitivo y esfuerzo de procesamiento. Bajo esta teoría, la relevancia es una noción de costo-beneficio (el costo corresponde al esfuerzo mental requerido y los beneficios a los efectos cognitivos conseguidos), y la tendencia universal hacia el incremento de la eficiencia de un sistema cognitivo mediante la maximización de la relevancia encaja perfectamente con la perspectiva evolutiva expuesta en la sección anterior. Esta tendencia es descrita en el primer principio cognitivo de la relevancia

(4) La cognición humana se orienta hacia la maximización de la relevancia. 
Se sigue de este principio que la atención humana y los recursos de procesamiento tienden automáticamente a asignarse a la información que parece ser lo suficientemente relevante para valer la pena su procesamiento. Esto no se debe a que conscientemente elijamos esta opción, sino que a la forma en que nuestros sistemas cognitivos han evolucionado.

As a result of constant selection pressure towards increasing efficiency, the human cognitive system has developed in such a way that our perceptual mechanisms tend automatically to pick out potentially relevant stimuli, our memory retrieval mechanisms tend automatically to activate potentially contextual assumptions, and our inferential mechanisms tend spontaneously to process them in the most productive way. It is against this cognitive background that communication takes place (Wilson, 2005:1145).

Sabiendo que es probable que los demás pongan atención a los estímulos más relevantes de su ambiente y a procesar aquellos de tal forma de maximizar su relevancia, las personas son capaces de producir estímulos los cuales probablemente atraerán la atención de los demás. Según la teoría de la relevancia (Sperber y Wilson, 1995, 2004), la comunicación ostensiva involucra dos tipos de intenciones:

(5) Intención informativa: La intención de informar a la audiencia respecto de algo.

(6 Intención comunicativa: La intención de informar a la audiencia de la propia intención informativa.

¿Cómo el hablante indica a su audiencia que él está tratando de comunicarse con ellos en esta forma manifiesta e intencional? La teoría de la relevancia sostiene que la comunicación manifiesta involucra el uso de un estímulo ostensivo, designado para atraer la atención de la audiencia sobre el significado del hablante. De esta forma, la comunicación se sirve del principio general de relevancia de la cognición, conformándose así el segundo principio de la relevancia, en este caso de índole comunicativa. De acuerdo con este segundo principio, el uso de un estímulo ostensivo activa una expectativa de relevancia a nuestros oyentes que, de otro modo, bajo las acciones o conductas ordinarias no comunicativas, no conllevaría.

(7) Principio comunicativo de relevancia: Cada enunciado (o cualquier acto de comunicación manifiesta) comunica la presunción de su propia relevancia óptima. 
Se sigue de este principio que el hablante, con el mero acto de emitir un enunciado a otro, comunica que su enunciado es el más relevante o compatible con las preferencias del oyente y que al menos es lo suficientemente relevante como para que valga la pena procesarlo.

La regularidad de la comunicación ostensiva descrita en el principio comunicativo de la relevancia motiva un proceso de comprensión (mediante la construcción de inferencias) de propósitos especiales el cual es automáticamente aplicado al procesamiento en-línea de los inputs verbales. El oyente toma el significado lingüístico decodificado; siguiendo el camino de menor esfuerzo de procesamiento, utiliza la información contextual disponible para enriquecer el nivel explícito del enunciado y para complementar el nivel implícito hasta que la interpretación satisfaga su expectativa de relevancia (Sperber y Wilson, 2004; 1995).

El procedimiento de comprensión guiado por la teoría de la relevancia es de carácter heurístico, rápido y parsimonioso. Es decir, es un proceso inferencial de propósitos especiales justificado por la regularidad descrita en el principio comunicativo de la relevancia, el cual permite generar conclusiones confiables solo cuando es aplicado a los inputs del dominio de la comunicación ostensiva (Sperber y Wilson, 2002; Wilson, 2005). En general, a un observador no le corresponde esperar que las acciones no comunicativas de los otros sean lo suficientemente relevantes como para merecer su atención. Sin embargo, cuando interpreta enunciados, al oyente le corresponde tal expectativa (debido al principio comunicativo de la relevancia). El procedimiento de comprensión de la teoría de la relevancia corresponde a la construcción automática de una hipótesis del significado del hablante sobre la base de la descripción del enunciado más la información contextual disponible, de la misma manera en que el Detector de Dirección de la Mirada automáticamente construye una hipótesis respecto de lo que un individuo está observando sobre la base de la dirección de su mirada (Bara, 2010; Tomasello, 2003). Bajo esta perspectiva, las regularidades descritas en los principios cognitivos y comunicativos de la relevancia proveen una base adecuada para un mecanismo de comprensión dedicado a la comunicación ostensiva, un submódulo del módulo de la teoría de la mente.

\section{Conclusiones}

El aporte de la presente investigación consiste en aplicar criterios de evaluación de teorías pragmáticas basadas en las consideraciones biológicas dentro de un marco evolucionista (Barkow, Cosmides y Tooby, 1992). En la presente investigación se defiende y argumenta en favor de una perspectiva cognitiva de la pragmática y se propone su rol funcional en el marco de una arquitectura modular masiva de la mente. En este sentido, proponemos que una teoría respecto del rol funcional de la pragmática en la arquitectura mental debe ser consistente con toda la evidencia empírica en torno a cómo las habilidades pragmáticas operan en 
términos cognitivos. A su vez, una buena teoría pragmática debe ser coherente con el marco de la psicología evolutiva. De acuerdo con esta última idea, las explicaciones de nuestras funciones cognitivas, como las habilidades pragmáticas, requiere que dichas funciones sean explicadas en virtud de cómo ellas están organizadas para satisfacer las demandas que las presiones ambientales ejercen sobre los seres vivos. Ello porque no solamente se debe exigir a una teoría que posea coherencia interna y manifestación empírica, sino que también ella debe poseer una consistencia con los demás campos científicos adyacentes. Bajo la revisión de la evidencia empírica (Airenti, 2017; Bara, 2010; Cummings, 2009; Happé, 1993; Papafragou, 2002; Tomasello, 2003) y un análisis de consistencia argumental, la teoría de la pragmática modular y de la modularidad masiva cumplen con tales exigencias. Bajo el análisis de las investigaciones empíricas sobre las habilidades pragmáticas y las consideraciones evolutivas se propone que el modelo que mejor encaja y satisface estos requerimientos corresponde a la perspectiva de la modularidad masiva de la mente, entendiendo la pragmática como un submódulo de habilidades de Teoría de la Mente. Dicha organización es consistente con los resultados observados en personas con déficit pragmático y además es coherente con respecto a consideraciones evolutivas.

La propuesta compartida en esta investigación es de carácter intuitiva y si bien existen antecedentes empíricos que la apoyan (sobre todo respecto de estudios de trastornos neurológicos y del desarrollo), todavía requiere ser desarrollada con más exhaustividad. Uno de los problemas que una perspectiva de la pragmática modular debe resolver, en el contexto de la modularidad masiva, se refiere a cómo se accede a las representaciones contextuales que se requieren para realizar una interpretación pragmática. Dado que no existe una restricción en principio en el tipo de fuente de información contextual que es utilizada en la construcción de una hipótesis respecto del significado pretendido por el hablante, el proceso sería global en el sentido fodoriano. De cualquier forma, no se sigue de todo esto que toda la información contextual tenga que estar activamente procesada para que la interpretación se lleve a cabo. El procedimiento de comprensión de la teoría de la relevancia sigue el camino de menor esfuerzo, utilizando cualquier tipo de información contextual que esté altamente activada mediante el trabajo automático de un sistema cognitivo en un momento dado. Sin embargo, para dar cuenta de la docilidad computacional que se requiere, es indispensable que se estipule de una manera sencilla cómo el módulo pragmático accede a información contextual sin que ocurra una explosión computacional. Una respuesta tentativa es que la presentación del estímulo comunicativo activa en-línea (online) una serie de representaciones en los mecanismos de memoria, las cuales son accesibles por parte del módulo pragmático. De todas formas, esta última hipótesis debe ser desarrollada con más profundidad. 
Por otra parte, es necesario también enfatizar que, en el caso de que se asuma que la pragmática es un sistema que funciona de manera rápida, automática y con sus propios principios y procedimiento, la pregunta que se sigue es ¿cuál es el dominio de este módulo? La respuesta diferencia aún más la propuesta de Sperber y Wilson con la de Asa Kasher. El dominio del módulo pragmático, según Sperber y Wilson, no lo constituyen únicamente los estímulos lingüísticos, sino que cualquier hecho comunicativo ostensivo. La teoría de la relevancia sostiene que la pragmática corresponde a un dominio más amplio que al de la conducta lingüística. Su dominio se vincula a los estímulos ostensivos de carácter comunicativo, sean estos basados en un código lingüístico o no. 
100 - Cogency, Journal of reasoning and argumentation

\section{Bibliografía}

Austin, J. (1998). Cómo hacer cosas con las palabras. Barcelona: Paidós.

Bara, B. (2010). Cognitive pragmatics: the mental processes of communication. Massachusetts: MIT Press.

Barker-Plummer, D. (22 de Junio de 2003). The Stanford Encyclopedia of Philosophy. Obtenido de The Stanford Encyclopedia of Philosophy: http://plato.stanford.edu/ archives/sum2013/entries/turing-machine/

[Consulta 02/05/2021]

Barkow, J., Cosmides, L., y Tooby, J. (1992). The adapted mind: Evolutionary psychology and the generation of culture. Oxford: Oxford Univesity Press.

Baron-Cohen, S. (1997). Mindblindness. Nueva York: A Bradford Book.

Barsalou, L. (2010). Grounded cognition: Past, present, and future. Topics in cognitive science, 2(4): 716-724.

Bloom, P. (2002). Mindreading, Communication and the Learning of Name for Things. Mind and Language, 17(1): 37-54.

Carruthers, P. (2006). The case for massively modular models of mind. En R. Stainton (eds.), Contemporary debates in cognitive science pp. 3-21. Oxford: Blackwell Publishing.

Carruthers, P., y Smith, P. (1996). Theories of Theories of Mind. Cambridge: Cambridge University Press.

Carston, R., y Powell, G. (2008). Relevance theory-new directions and developments. En E. Lepore, y B. Smith, The Oxford Handbook of Philosophy of Language, pp. 341360. Oxford: Oxford University Press.

Chomsky, N. (1966). Cartesian Linguistics: A Chapter in the History of Rationalist . Nueva York: Harper y Row.

Chomsky, N. (1980). Rules and Representations. Nueva York: Columbia University Press.

Cummings, L. (2005). Pragmatics a multidisciplinary perspective. Nueva Jersey: Lawrence Erlbaum Associates.

Cummings, L. (2009). Clinical Pragmatics. Cambridge: Cambridge University Press.

Desai, R., Binder, J., Conant, L., y Seidenberg, M. (2010). Activation of sensory-motor areas in sentence comprehension. Cerebral Cortex, 20(2): 468-478. 
Fischer, M., y Zwaan, R. (2008). Embodied language: A review of the role of the motor system in language comprehension. Quarterly journal of experimental psychology, 61(6): 825-850.

Fodor, J. (1980). The Language and Thought. Harvard University Press: Cambridge.

Fodor, J. (1983). The modularity of the mind. Cambridge: MIT Press.

Fodor, J. (2000). The mind doesn't work that way. Cambridge: MIT Press.

Grice, P. (1975). Logic and Conversation. En P. Cole, y J. Morgan (eds.), Syntax and Semantics Vol. 3: Speech Acts, pp. 41-58. Nueva York: Academic Press.

Grice, P. (1989). Studies in the Way of Words. Cambridge: Harvard University Press.

Happé, F. (1993). Communicative competence and theory of mind in autism: A test of relevance theory. Cognition, 48: 101-119.

Happé, F., y Loth, E. (2002). Theory of Mind and Tracking Speakers Intentions. Mind and Language, 17: 24-36.

Kasher, A. (1991). Pragmatics and the modularity of the mind. En S. Davis (ed.), Pragmatics a reader, pp. 567-582. Oxford: Oxford University Press.

Leech, G. (1983). Principles of Pragmatics. Londres: Logman.

Mazzarella, D. (2016). Pragmatics, modularity and epistemic vigilance. Argumenta, 1(2): 181-193.

Mazzarella, D., y Pouscoulous, N. (2021). Pragmatics and epistemic vigilance: A developmental perspective. Mind and Language, 36(3): 355-376.

Ostarek, M., y Huettig, F. (2017). Spoken words can make the invisible visible-Testing the involvement of low-level visual representations in spoken word processing. Journal of Experimental Psychology: Human Perception and Performance, 43(3): 499.

Papafragou, A. (2002). Mindreading in verbal communication. Mind y Language, 17: 55-67.

Searle, J. (1975). Una taxonomía de los actos ilocucionarios. En L. Valdés Villanueva (ed.), La búsqueda del significado, pp. 328-339. Madrid: Paidós.

Segal, G. (1996). The modularity of theory of mind. En P. Carruthers, y P. Smith (eds.), Theories of Theories of Mind, pp. 141-157. Cambridge: Cambridge University Press.

Simon, H. (1962). The architecture of complexity. Proceedings of the American Philosophical Society, 106: 467-482. 
Sperber, D. (2000). Metarepresentations in a evolutionary perspective. En D. Sperber (ed.), Metarepresentations: A Multidisciplinary Perspective, pp. 117-137. Oxford: Oxford University Press.

Sperber, D. (2002). In Defense of Massive Modularity. En E. Dupoux (ed.), Language, Brain, and Cognitive Development: Essays in Honor of Jacques Mehler, pp. 47-58. Oxford: A Bradford Book.

Sperber, D., y Wilson, D. (1995). Relevance. Cambridge: Blackwell.

Sperber, D., y Wilson, D. (2002). Pragmatics, Modularity and Mind-Reading. Mind y Language, 17: 3-23.

Sperber, D., y Wilson, D. (2004). Relevance Theory. En L. Horn, y G. Ward (eds.), The Handbook of Pragmatics pp. 607-632. Oxford: Blackwell Publishing.

Stillings, N., Weisler, S., Chase, C., Feinstein, M., Garfield, J., y Rissland, E. (1995). Cognitive Science. An Introduction. Cambridge: Bradford.

Tager-Flusberg, H. (1999). Neurodevelopmental Disorders. Cambridge: MIT Press.

Thagard, P. (2012). Cognitive architectures. En K. Frankish, y W. Ramsey (eds.), The Cambridge Handbook of Cognitive Science, pp. 50-71. Cambridge: Cambridge University Press.

Tomasello, M. (2003). Constructing a Language: a Usage-Based Theory of Language Acquisition. Cambridge: Harvard University Press.

Varley, R. (2002). Science without grammar: scientific reasoning in severe agrammatic aphasia. En P. Carruther, S. Stich, y M. Siegal (eds.), The cognitive Basis of Science, pp. 99-116. Cambridge: Cambridge University Press.

Von Eckardt, B. (2012). The Representational Theory of Mind. En K. Frankish, y W. Ramsey (eds.), The Cambridge Handbook of Cognitive Science, pp. 29-49. Cambridge: Cambridge University Press.

Wilson, D. (2000). Metarepresentation in linguistic communication. En D. Sperber (ed.), Metarepresentations: An interdisciplinary Perspective, pp 411-450. Nueva York: Oxford University Press.

Wilson, D. (2005). New directions for research on pragmatics and modularity. Lingua, 115: 1129-1146. 